\title{
Materials Informatics Approach Using Domain Modelling For Exploring Structure-Property Relationships Of Polymers
}

\author{
Koki Hara \\ Yokohama City University \\ Shunji Yamada \\ RIKEN Center for Sustainable Resource Science \\ Atsushi Kurotani \\ RIKEN Center for Sustainable Resource Science \\ Eisuke Chikayama \\ Niigata University of International and Information Studies \\ Jun Kikuchi ( $\nabla$ jun.kikuchi@riken.jp ) \\ RIKEN Center for Sustainable Resource Science
}

\section{Research Article}

Keywords:

Posted Date: February 28th, 2022

DOI: https://doi.org/10.21203/rs.3.rs-1361413/v1

License: (c) (1) This work is licensed under a Creative Commons Attribution 4.0 International License. Read Full License 


\section{Abstract}

In the development of polymer materials, it is an important issue to explore the complex relationships between domain structure and physical properties. In the domain structure analysis of polymer materials,

${ }^{1} \mathrm{H}$-static solid-state NMR (ssNMR) spectra can provide information on mobile, rigid, and intermediate domains. But estimation of domain structure from its analysis is difficult due to the wide overlap of spectra from multiple domains. Therefore, we have developed a materials informatics approach that combines the domain modeling (http://dmar.riken.jp/matrigica/) and the integrated analysis of metainformation (the elements, functional groups, additives, and physical properties) in polymer materials. Firstly, the ${ }^{1} \mathrm{H}$-static ssNMR data of 120 polymer materials were subjected to a short-time Fourier transform to obtain frequency, intensity, and $T_{2}$ relaxation time for domains with different mobility. The average $T_{2}$ relaxation time of each domain is $0.96 \mathrm{~ms}$ for Mobile, $0.55 \mathrm{~ms}$ for Intermediate (Mobile), 0.32 $\mathrm{ms}$ for Intermediate (Rigid), and $0.11 \mathrm{~ms}$ for Rigid. Secondly, the estimated domain proportions were integrated with meta-information such as elements, functional group and thermophysical properties and was analyzed using a self-organization map and market basket analysis. This proposed method can contribute to explore structure-property relationships of polymer materials with multiple domains.

\section{Introduction}

Traditional design approaches for materials are experimentally driven, facing significant challenges due to the vast design space of materials. Experimental science can be supported by materials informatics ${ }^{1-3}$ that makes full use of theoretical and computational science such as density functional theory (DFT $)^{4,5}$ and molecular dynamics (MD) ${ }^{6}$ and data science using computers (Artificial Intelligence; Al) ${ }^{7,8}$. Computational science solves equations numerically based on theory and physical models. On the other hand, data science explores candidate materials with a certain function from a large quantity of material data. Its physical meaning needs to be verified by experimental, theoretical and computational science. The meta-information involved in the production process of the material also plays an important role in desired materials development ${ }^{9,10}$.

In recent years, the development of sustainable polymers that meet the needs of consumers without destroying the environment has become an important issue due to global problems such as marine pollution, waste disposal, and global warming caused by plastics. ${ }^{11,12}$ Furthermore, "carbon-neutral" biobased polymers, such as polylactic acid (PLA), polybutylene succinate (PBS), poly(3-hydroxybutyrate-co3-hydroxyhexanoate) (PHBH), and poly- $\varepsilon$-caprolactone (PCL), have become a focus in the era of biorefinery materials as an alternative to oil-based materials ${ }^{13,14}$. Polymers such as $\mathrm{PLA}^{15}, \mathrm{PCL}^{16}$, are multiple domain systems, are often employed as high-performance materials which can display various properties.

Solid-state nuclear magnetic resonance (ssNMR) spectroscopy is a powerful tool that is used to characterize the native structure, components and dynamics of solid-state samples at the atomic level, 
and has been increasingly applied in material sciences ${ }^{17,18}$. In addition, NMR measurements, especially low magnetic field NMR, is a method for routine material evaluations, which has produced a lot of NMR datasets $^{19}$. Typical ssNMR methods are cross-polarization (CP)/magic-angle spinning (MAS) methods with elimination of linewidth broadening due to chemical shift anisotropy for high resolution. On the other hand, there are different complementary approaches to tackle complexity of polymer domain structure. ${ }^{1} \mathrm{H}$-static ssNMR can be applied to quantify domain mobility in terms of dynamic heterogeneity. ${ }^{20} \mathrm{In}$ addition, the use of magic-and-polarization echo (MAPE) ${ }^{21}$ and double-quantum (DQ) ${ }^{22}$ filters can determine the spectral parameters for the mobile amorphous domains with the long-time decay and the strongly dipole-dipole-coupled crystalline domains with the quickly decay, respectively.

In the case of characterization of a solid-state sample with domains of rigid, intermediate and mobile types, the ${ }^{1} \mathrm{H}$-static ssNMR measurement is useful as a measure of the kinetic nature of higher order structures, although its analysis is difficult because the spectrum is broadened and overlapped ${ }^{23}$. Therefore, the application of signal deconvolution to measured ssNMR data is an important challenge as one of computational approaches to measured data. Several methods for spectral separation ${ }^{19}$, fitting and numerical simulation ${ }^{24}$ such as SIMPSON ${ }^{25}$, SPINEVOLUTION ${ }^{26}$, dmfit $^{27}$, EASY-GOING deconvolution ${ }^{28}$, INFOS ${ }^{29}$, Fityk $^{30}$, ssNake ${ }^{31}$, and a noise reduction method based on principal component analysis ${ }^{32}$ have been developed. So far, in NMR data analysis, signal simulation and fitting have targeted only the frequency domain or the time domain. In our previous study, we proposed signal deconvolution methods that combines short-time Fourier transform (STFT; a time-frequency analytical method) and probabilistic sparse matrix factorization ${ }^{33}$, and non-negative tensor/matrix factorization ${ }^{34}$. In our method using STFT, by simulating the signal for both the frequency and time domain, it was possible to separate the signal related to the motility characteristics of the domain structure based on the indicators of chemical shift and $T_{2}$ relaxation time. The NMR signal can be calculated by functions such as Lorentzian ${ }^{35}$, Gaussian ${ }^{36}$, and Voigt ${ }^{37}$ in the frequency domain, and by the $T_{2}$ relaxation Eq. $3^{8}$ in the time domain. In addition, the difference in $T_{2}$ relaxation times can be adjusted by the Weibull coefficient ${ }^{39}$. Analysis of the relaxation time of a sample's free-induction decay (FID) provides important insights into the chemical composition, structure, and mobility of the sample ${ }^{38,40}$.

In this study, we propose a materials informatics approach to explore the structure-property relationships of polymers that combines the polymer domain modeling and the integrated analysis of polymer materials meta-information. For polymer domain modeling, ${ }^{1} \mathrm{H}$-static ssNMR spectral parameters obtained using STFT were utilized, including $T_{2}$ relaxation time, frequency, and intensity. The domain structure with different mobility in the polymeric material was estimated by fitting the physical indices such as $T_{2}$ relaxation time, frequency, and linewidth. In addition, using a self-organization map (SOM) and market basket analysis (MBA), the relationships between the estimated domain structure and the meta-information such as elements, functional group, and thermophysical property were explored.

\section{Results And Discussion}




\section{A Materials Informatics Approach to Exploring Structure-Property Relationships Using Domain Modeling.}

The conceptual diagram of materials informatics approach to exploring structure-property relationships using domain modeling is shown in the Fig. 1. The detailed analytical flow of this method is shown in Supporting Information Figure S1. We have utilized the input polymer information that are ${ }^{1} \mathrm{H}$-static ssNMR ( ${ }^{1} \mathrm{H}$-static ssNMR) data, primary structure of the polymer, and thermophysical property data (TG/DTA/DTG). Then, frequency and time information are obtained by STFT against FIDs obtained by ${ }^{1} \mathrm{H}$-static ssNMR. The domain modeling method is the following. The domain components are firstly separated by fitting the obtained frequency and time information (Figure S2). Secondly, the domain component ratio is calculated by 3D modeling (Figure S3). After that, we performed statistical analysis, MBA, and SOM, which are materials informatics methods, on the obtained domain information, primary structure information, and thermophysical property information to associate the structure and physical properties. The detailed results are shown in the following sections.

\section{Results of Domain Modeling of Polymer Materials Using Time-Frequency Simulation of ${ }^{1} \mathrm{H}$-Static ssNMR Spectra.}

The ratios of the domain components were calculated from the volume ratios obtained from domain modeling of 71 samples of the polymer materials (Fig. 2). We have classified analyzed data in this study into four domain components: Mobile, Intermediate (Mobile), Intermediate (Rigid), and Rigid domain components, those we regard to mobile, slightly mobile, slightly rigid, and rigid components in their material states, respectively. As a result, the domain component ratios indicated differences among not only different polymer materials but also similar ones that composed of the same monomers, which can be attributed to the molding conditions and molecular weight. In the case of PCL (Fig. 2, upper right), which is the sample with the most Mobile domain component: the Mobile domain component ratio was $37.7 \%$; the Intermediate (Mobile) domain component ratio was $11.2 \%$; the Intermediate (Rigid) domain component ratio was $18.1 \%$; and the Rigid domain component ratio was $33.0 \%$. In the case of $\mathrm{PHBH}$ sample (Fig. 2, upper left), which has the highest Rigid domain component: the Mobile domain component ratio was $10.4 \%$; the Intermediate (Mobile) domain component ratio was $3.1 \%$; the Intermediate (Rigid) domain component ratio was $36.5 \%$; and the Rigid domain component ratio was $50.5 \%$. From this result, we were able to calculate the difference in domain ratios caused by the different monomers used to synthesize the polymer.

The calculated distributions of $T_{2}$ relaxation time and thermophysical properties of the domain components of each polymer are shown (Fig. 3). The box-and-whisker plot shows that the average $T_{2}$ relaxation time information is $0.96 \mathrm{~ms}$ for Mobile, $0.55 \mathrm{~ms}$ for Intermediate (Mobile), $0.32 \mathrm{~ms}$ for Intermediate (Rigid), and $0.11 \mathrm{~ms}$ for Rigid. The weighted average (WA) of the polymer materials showed a distribution among the samples, among which PCL (Fig. 3, red squares) was high and PHBH (Fig. 3, yellow circles) was low. The same was true for the results of thermal analysis spectral data (Table S3). Based on the domain component ratios, the estimated domain ratio diagrams were inserted for the highest PCL and lowest PHBH of WA. 

Materials.

In order to evaluate the relationships between domain structure and thermophysical properties of polymer materials, we integrated the domain proportion described in the previous section, ${ }^{13} \mathrm{C}-\mathrm{CP} / \mathrm{MAS}$ spectra ${ }^{41}$, which easily reflect primary chemical structure, and quantitative thermophysical data (thermogravimetry (TG), differential thermal analysis (DTA), derivative thermogravimetry (DTG), differential scanning calorimetry (DSC) $)^{42}$. To capture the characteristics of the integrated data, clustering by SOM was performed (Fig. 4). These materials clustered in the following way i.e., navy blue circle symbols are polyethylene terephthalate (PET), light blue circle symbols are polyethylene (PE), and the clusters of these polymer materials are on the top, blue triangle symbols are poly(butylene adipate-co-terephthalate) (PBAT), light pink circle symbols are polylactic acid (PLA), light green circle symbols are poly(hexano-6lactam) (nylon), and the clusters of these polymer materials are on the bottom left, black cross symbols are polybutylene succinate (PBS), orange diamond symbols are poly(butylene succinate-co-butylene adipate) (PBSA), and red square symbols are PCL, and the clusters of these polymer materials are found to exist solidly in the lower right corner, respectively. These clusters formed two groups: high heat resistant polymers with many rigid domain components, and low heat resistant polymers with many mobile domain components ${ }^{43,44}$. The results of thermal analysis for PET, PE, PBAT, PLA, nylon, PBS, PBSA, and PCL were consistent with their characteristics shown above (Figure S4, Table S3).

\section{Market Basket Analysis Integrating Quantitative Domain Proportion and Qualitative Meta-Information in Polymer Materials.}

The MBA was performed to evaluate the relationships between the quantitative domain proportion as well as qualitative meta-information such as elements (linearly connected methylenes $>4$ carbons), functional groups (aromaticity), and thermophysical properties (Table S3). Using the transaction data based on the MBA, a network diagram is shown in Fig. 5, where the $T_{2}$ information of the four domain components (Mobile, Intermediate (Mobile), Intermediate (Rigid), and Rigid) shows a high lift value with the primary structure information (aromaticity, linearly connected methylenes $>4$ carbons). While the thermophysical properties (melting temperature, $T_{\text {m }}$; thermal decomposition temperature, $T_{\mathrm{d}}$; glass transition temperature, $T_{\mathrm{g}}$ ) show a dominant lift value with their structure information. The lift value here is one of the indicators for correlation analysis in MBA. Figure S5 shows the MBA network diagram using the temperature information from thermal analysis, where the Mobile domain ratio correlated with the lower temperature $\left(<100^{\circ} \mathrm{C}\right)$ of thermophysical properties. The Intermediate (Mobile) domain ratio from the MAPE Filter and the Intermediate (Rigid) domain ratio from the DQ Filter showed intermediate thermophysical properties $\left(100\right.$ to $\left.300^{\circ} \mathrm{C}\right)$. The rigid domain ratio correlated with the lower temperature $(<$ $100^{\circ} \mathrm{C}$ ) and of the higher temperature $\left(>300^{\circ} \mathrm{C}\right)$ thermophysical properties. In common, PCL is a thermoplastic biodegradable polyester with good thermal processability and low melting point ${ }^{43}$. In PCL, there is a melting temperature at $66.5^{\circ} \mathrm{C}$ of DTG (Figure S4, Table S3). While the DTG peak of lower temperature is correlated with the Rigid domain (Figure S5a). 


\section{Conclusion}

In the development of materials, in addition to the chemical structure from the primary to the higher-order, meta-information such as molding process and additives are important factors because these have great influences on the final material properties. We have developed a materials informatics approach that combines the domain modeling and the integrated analysis of materials meta-information. To estimate the domain structure information, we have introduced a time-frequency simulation method for calculating multiple domain components from ${ }^{1} \mathrm{H}$-static ssNMR spectra. In our integrated analysis of domain proportions and meta-information, SOM was a useful tool for capturing trends across polymer material data. On the other hand, MBA was able to investigate the strong relationships between structure and meta information in individual materials, including qualitative data as well as quantitative data. The relationships between mobility of domain structure and melting temperature were similar to the results shown by SOM and MBA (Fig. 3, 4, 5). ${ }^{43,45}$ This materials informatics approach is expected to efficiently explore relationships between structure and properties of high-performance and low environmental impact polymer materials.

\section{Materials And Methods}

\section{Materials.}

Polymer materials (Table S1) were prepared using a press molding machine (H300-01, AS ONE Corp., Osaka, Japan) and molding methods reported in a previous study 46 .

\section{Time-Frequency Simulation of ${ }^{1} \mathrm{H}$-static ssNMR spectra.}

The time-frequency simulation method was developed in Python 3, by using the packages of nmrglue ${ }^{47}$ for processing of NMR data, Scipy.signal for the Fourier transform, STFT, and mathematical processing, the curve-fit function of scipy.optimize and BayesianOptimization for the fitting process, and mpl_toolkits for visualization of 3D (time, frequency, and intensity) simulation model. Before applying this method, the FID data was phase-corrected, baseline-corrected, and inverse Fourier transformed using TopSpin (BrukerBioSpin, MA, USA). In order to calculate the ratio between the Mobile and Intermediate (Mobile) domain components obtained from the MAPE filtered spectra, and the Rigid and Intermediate (Rigid) domain components obtained from the DQ filtered spectra, the calculation errors between the four domain components and the STFT ${ }^{1} \mathrm{H}$-static spectra (Static) were calculated using the following equation (Eq. (1)).

$$
\text { Calculationerror }=\text { Static }-\left(\alpha \times\left(M_{\text {Mobile }}+M_{I M}\right)+\beta \times\left(M_{I R}+M_{\text {Rigid }}\right)\right)
$$


After finding the $\alpha$ and $\beta$ parameters that minimize the error, we created a 3D model of the four domain components based on the frequency and $T_{2}$ relaxation time information.

The domain component ratios contained in the polymer material were calculated using the following equation (Eq. (2-5)).

$$
M_{\text {Mobile }}=\frac{M(t) L(x)}{M_{0}^{\prime}}
$$

2

$$
M_{I M}=\frac{M(t) I M(x)}{M_{0}^{\prime}}
$$

3

$$
M_{I R}=\frac{M(t) I R(x)}{M_{0}^{\prime}}
$$

4

$$
M_{\text {Rigid }}=\frac{M(t) G(x)}{M_{0}^{\prime}}
$$

5

A detailed description of the time-frequency simulation method is given in Supporting Information Figure S1 and Figure S2.

The weighted average (WA) of the $T_{2}$ relaxation times for a single polymer material was calculated using the following equation (Eq. (6)).

$$
\begin{gathered}
W A=\left\{M_{\text {Mobile }} T_{2}(\text { Mobile })+M_{I M} T_{2}^{I M}+M_{I R} T_{2}^{I R}\right. \\
\left.+M_{\text {Rigid }} T_{2}(\text { Rigid })\right\} /\left(M_{\text {mobile }}+M_{I M}+M_{I R}+M_{\text {Rigid }}\right)
\end{gathered}
$$

6

\section{Integrated Analysis of Domain Proportions and Meta-Information in Polymer Materials.}

In the integrated analysis of domain proportions and meta-information of polymer materials, a $\mathrm{SOM}^{48}$ was produced using the $\mathrm{R}$ package kohonen, and $\mathrm{MBA}^{49}$ was performed using the $\mathrm{R}$ package arules. The association network was visualized using the Cytoscape program. 


\section{Tool Development for Automated Spectral Simulation.}

We have created a Bayesian optimization-based ${ }^{50}$ spectral simulation tool that automates the $T_{2}$ relaxation time domain fitting, frequency fitting, and 3D domain modeling of our domain component separation method. The details of the Python program including $T_{2}$ relaxation time information, frequency information, and 3D domain modeling of the present domain component separation method can be obtained at https://github.com/riken-emar/matrigica. For improving the level of accuracy of the prediction, intermediate regression models were employed when performing in-phase machine learning. In addition, we developed a website dedicated to the established domain component ratio calculation, which is freely available at http://dmar.riken.jp/matrigica/.

\section{Declarations}

\section{Author contributions}

The manuscript was written through contributions of all authors. All authors have given approval to the final version of the manuscript. K.H., S.Y, E.C, and J.K designed this study. Analytical flow and python tools were developed by K.H., S.Y. and A.K.

\section{Data availability}

The analytical tool and numerical data for association analysis used in this study were deposited on the following websites: http://dmar.riken.jp/NMRinformatics/MatRigiCa.zip and http://dmar.riken.jp/NMRinformatics/DatasetForMatRigiCa.zip.

\section{Competing interests}

The authors declare no competing interests.

\section{Acknowledgements}

The authors thank Yuuri Tsuboi, Tomoko Matsumoto, Akiyo Tei (RIKEN) for their support with NMR data acquisition and handling. S.Y. was supported by the RIKEN Junior Research Associate Program and the promotion program for informatics and data science of RIKEN Center for Sustainable Resource Science during the part of a period of this research. This work was partially supported by a grant from the Agriculture, Forestry, and Fisheries Research Council, as well as Strategic Innovation Program (SIP) from CAO of Japan (to J.K.).

\section{References}

1. Ben-Sasson, A. J. et al. Design of biologically active binary protein $2 \mathrm{D}$ materials. Nature $\mathbf{5 8 9}, \mathbf{4 6 8}$ 473, doi:10.1038/s41586-020-03120-8 (2021). 
2. Jha, D. et al. Enabling deeper learning on big data for materials informatics applications. Sci Rep 11, 4244, doi:10.1038/s41598-021-83193-1 (2021).

3. Ramprasad, R., Batra, R., Pilania, G., Mannodi-Kanakkithodi, A. \& Kim, C. Machine learning in materials informatics: recent applications and prospects. npj Comput. Mater. 3, 13, doi:10.1038/s41524-017-0056-5 (2017).

4. Ito, K., Xu, X. \& Kikuchi, J. Improved Prediction of Carbonless NMR Spectra by the Machine Learning of Theoretical and Fragment Descriptors for Environmental Mixture Analysis. Analytical Chemistry 93, 6901-6906, doi:10.1021/acs.analchem.1c00756 (2021).

5. Ito, K., Obuchi, Y., Chikayama, E., Date, Y. \& Kikuchi, J. Exploratory machine-learned theoretical chemical shifts can closely predict metabolic mixture signals. Chemical Science 9, 8213-8220, doi:10.1039/c8sc03628d (2018).

6. Mori, T. et al. Exploring the conformational space of amorphous cellulose using NMR chemical shifts. Carbohydr Polym 90, 1197-1203, doi:10.1016/j.carbpol.2012.06.027 (2012).

7. Himanen, L., Geurts, A., Foster, A. \& Rinke, P. Data-Driven Materials Science: Status, Challenges, and Perspectives (vol 6, 1900808, 2019). Advanced Science 7, doi:10.1002/advs.201903667 (2020).

8. Chen, G. et al. Machine-Learning-Assisted De Novo Design of Organic Molecules and Polymers: Opportunities and Challenges. Polymers 12, doi:10.3390/polym12010163 (2020).

9. Ma, R. \& Luo, T. PI1M: A Benchmark Database for Polymer Informatics. J Chem Inf Model 60, 46844690, doi:10.1021/acs.jcim.0c00726 (2020).

10. Granda, J. M., Donina, L., Dragone, V., Long, D. L. \& Cronin, L. Controlling an organic synthesis robot with machine learning to search for new reactivity. Nature 559, 377-381, doi:10.1038/s41586-0180307-8 (2018).

11. Borrelle, S. B. et al. Predicted growth in plastic waste exceeds efforts to mitigate plastic pollution. Science 369, 1515-t, doi:10.1126/science.aba3656 (2020).

12. Kubowicz, S. \& Booth, A. M. Biodegradability of Plastics: Challenges and Misconceptions. Environmental Science \& Technology 51, 12058-12060, doi:10.1021/acs.est.7b04051 (2017).

13. Mohanty, A. K., Vivekanandhan, S., Pin, J. M. \& Misra, M. Composites from renewable and sustainable resources: Challenges and innovations. Science $362,536-542$, doi:10.1126/science.aat9072 (2018).

14. Ragauskas, A. J. et al. The path forward for biofuels and biomaterials. Science 311, 484-489, doi:311/5760/484 [pii] 10.1126/science.1114736 (2006).

15. Inkinen, S., Hakkarainen, M., Albertsson, A. \& Sodergard, A. From Lactic Acid to Poly(lactic acid) (PLA): Characterization and Analysis of PLA and Its Precursors. Biomacromolecules 12, 523-532, doi:10.1021/bm101302t (2011).

16. Schaler, K., Achilles, A., Barenwald, R., Hackel, C. \& Saalwachter, K. Dynamics in Crystallites of Poly(epsilon-caprolactone) As Investigated by Solid-State NMR. Macromolecules 46, 7818-7825, doi:10.1021/ma401532v (2013). 
17. Eden, M. Editorial for the Special Issue on Solid-State NMR Spectroscopy in Materials Chemistry. Molecules 25, doi:10.3390/molecules25122720 (2020).

18. Kikuchi, J., Ito, K. \& Date, Y. Environmental metabolomics with data science for investigating ecosystem homeostasis. Prog Nucl Magn Reson Spectrosc 104, 56-88, doi:10.1016/j.pnmrs.2017.11.003 (2018).

19. Yamada, S. et al. InterSpin: Integrated Supportive Webtools for Low- and High-Field NMR Analyses Toward Molecular Complexity. Acs Omega 4, 3361-3369, doi:10.1021/acsomega.8b02714 (2019).

20. Bärenwald, R., Achilles, A., Lange, F., Ferreira, T. M. \& Saalwächter, K. Applications of Solid-State NMR Spectroscopy for the Study of Lipid Membranes with Polyphilic Guest (Macro)Molecules. Polymers (Basel) 8, doi:10.3390/polym8120439 (2016).

21. Demco, D. E., Johansson, A. \& Tegenfeldt, J. Proton spin diffusion for spatial heterogeneity and morphology investigations of polymers. Solid State Nuclear Magnetic Resonance 4, 13-38, doi:10.1016/0926-2040(94)00036-c (1995).

22. Buda, A. et al. Domain sizes in heterogeneous polymers by spin diffusion using single-quantum and double-quantum dipolar filters. Solid State Nuclear Magnetic Resonance 24, 39-67, doi:10.1016/S0926-2040(03)00020-1 (2003).

23. Schaler, K. et al. Basic principles of static proton low-resolution spin diffusion NMR in nanophaseseparated materials with mobility contrast. Solid State Nuclear Magnetic Resonance 72, 50-63, doi:10.1016/j.ssnmr.2015.09.001 (2015).

24. Schneider, H., Saalwachter, K. \& Roos, M. Complex Morphology of the Intermediate Phase in Block Copolymers and Semicrystalline Polymers As Revealed by H-1 NMR Spin Diffusion Experiments. Macromolecules 50, 8598-8610, doi:10.1021/acs.macromol.7b00703 (2017).

25. Bak, M., Rasmussen, J. \& Nielsen, N. SIMPSON: A general simulation program for solid-state NMR spectroscopy. Journal of Magnetic Resonance 147, 296-330, doi:10.1006/jmre.2000.2179 (2000).

26. Veshtort, M. \& Griffin, R. SPINEVOLUTION: A powerful tool for the simulation of solid and liquid state NMR experiments. Journal of Magnetic Resonance 178, 248-282, doi:10.1016/j.jmr.2005.07.018 (2006).

27. Massiot, D. et al. Modelling one- and two-dimensional solid-state NMR spectra. Magnetic Resonance in Chemistry 40, 70-76, doi:10.1002/mrc.984 (2002).

28. Grimminck, D. et al. EASY-GOING deconvolution: Automated MQMAS NMR spectrum on a model with analytical crystallite excitation efficiencies. Journal of Magnetic Resonance 228, 116-124, doi:10.1016/j.jmr.2012.12.012 (2013).

29. Smith, A. INFOS: spectrum fitting software for NMR analysis. Journal of Biomolecular Nmr 67, 7794, doi:10.1007/s10858-016-0085-2 (2017).

30. Wojdyr, M. Fityk: a general-purpose peak fitting program. Journal of Applied Crystallography 43, 1126-1128, doi:10.1107/S0021889810030499 (2010).

31. van Meerten, S., Franssen, W. \& Kentgens, A. ssNake: A cross-platform open-source NMR data processing and fitting application. Journal of Magnetic Resonance 301, 56-66, 
doi:10.1016/j.jmr.2019.02.006 (2019).

32. Kusaka, Y., Hasegawa, T. \& Kaji, H. Noise Reduction in Solid-State NMR Spectra Using Principal Component Analysis. Journal of Physical Chemistry a 123, 10333-10338, doi:10.1021/acs.jpca.9b04437 (2019).

33. Yamada, S., Kurotani, A., Chikayama, E. \& Kikuchi, J. Signal Deconvolution and Noise Factor Analysis Based on a Combination of Time-Frequency Analysis and Probabilistic Sparse Matrix Factorization. International Journal of Molecular Sciences 21, doi:10.3390/ijms21082978 (2020).

34. Yamada, S., Chikayama, E. \& Kikuchi, J. Signal Deconvolution and Generative Topographic Mapping Regression for Solid-State NMR of Multi-Component Materials. Int J Mol Sci 22, doi:10.3390/ijms22031086 (2021).

35. Sun, Y. C. \& Xin, J. Lorentzian peak sharpening and sparse blind source separation for NMR spectroscopy. Signal Image and Video Processing, doi:10.1007/s11760-021-02002-4.

36. Wang, F., Deng, Z., Yang, Z. \& Sun, P. Heterogeneous Dynamics and Microdomain Structure of HighPerformance Chitosan Film as Revealed by Solid-State NMR. The Journal of Physical Chemistry C 125, 13572-13580, doi:10.1021/acs.jpcc.1c01801 (2021).

37. Bruce, S. D., Higinbotham, J., Marshall, I. \& Beswick, P. H. An analytical derivation of a popular approximation of the Voigt function for quantification of NMR spectra. Journal of Magnetic Resonance 142, 57-63, doi:10.1006/jmre.1999.1911 (2000).

38. Besghini, D., Mauri, M. \& Simonutti, R. Time Domain NMR in Polymer Science: From the Laboratory to the Industry. Applied Sciences-Basel 9, doi:10.3390/app9091801 (2019).

39. Rossini, A. J. et al. Dynamic Nuclear Polarization Enhanced NMR Spectroscopy for Pharmaceutical Formulations. Journal of the American Chemical Society 136, 2324-2334, doi:10.1021/ja4092038 (2014).

40. Borsacchi, S. et al. Rubber-Filler Interactions in Polyisoprene Filled with In Situ Generated Silica: A Solid State NMR Study. Polymers 10, 10, doi:10.3390/polym10080822 (2018).

41. Spaccini, R., Todisco, D., Drosos, M., Nebbioso, A. \& Piccolo, A. Decomposition of bio-degradable plastic polymer in a real on-farm composting process. Chemical and Biological Technologies in Agriculture 3, doi:10.1186/s40538-016-0053-9 (2016).

42. Budrugeac, P. et al. The use of thermal analysis methods for predicting the thermal endurance of an epoxy resin used as electrical insulator. Journal of Thermal Analysis and Calorimetry, doi:10.1007/s10973-020-10156-5.

43. Zhong, Y., Godwin, P., Jin, Y. \& Xiao, H. Biodegradable polymers and green-based antimicrobial packaging materials: A mini-review. Advanced Industrial and Engineering Polymer Research 3, 2735, doi:10.1016/j.aiepr.2019.11.002 (2020).

44. Post, W., Kuijpers, L. J., Zijlstra, M., van der Zee, M. \& Molenveld, K. Effect of Mineral Fillers on the Mechanical Properties of Commercially Available Biodegradable Polymers. Polymers (Basel) 13, doi:10.3390/polym13030394 (2021). 
45. Oyama, T. et al. Biodegradable Compatibilizers for Poly(hydroxyalkanoate)/Poly(epsiloncaprolactone) Blends through Click Reactions with End-Functionalized Microbial Poly(hydroxyalkanoate)s. Acs Sustainable Chemistry \& Engineering 7, 7969-+, doi:10.1021/acssuschemeng.9b00897 (2019).

46. Yamawaki, R., Tei, A., Ito, K. \& Kikuchi, J. Decomposition Factor Analysis Based on Virtual Experiments throughout Bayesian Optimization for Compost-Degradable Polymers. Applied Sciences-Basel 11, doi:10.3390/app11062820 (2021).

47. Helmus, J. J. \& Jaroniec, C. P. Nmrglue: an open source Python package for the analysis of multidimensional NMR data. J Biomol NMR 55, 355-367, doi:10.1007/s10858-013-9718-x (2013).

48. Ito, K., Sakata, K., Date, Y. \& Kikuchi, J. Integrated Analysis of Seaweed Components during Seasonal Fluctuation by Data Mining Across Heterogeneous Chemical Measurements with Network Visualization. Analytical Chemistry 86, 1098-1105, doi:10.1021/ac402869b (2014).

49. Wei, F. F., Sakata, K., Asakura, T., Date, Y. \& Kikuchi, J. Systemic Homeostasis in Metabolome, Ionome, and Microbiome of Wild Yellowfin Goby in Estuarine Ecosystem. Scientific Reports 8, doi:10.1038/s41598-018-20120-x (2018).

50. Kurotani, A., Kakiuchi, T. \& Kikuchi, J. Solubility Prediction from Molecular Properties and Analytical Data Using an In-phase Deep Neural Network (Ip-DNN). ACS Omega 6, 14278-14287, doi:10.1021/acsomega.1c01035 (2021).

\section{Figures}

\section{Figure 1}

Conceptual diagram of materials informatics approach to exploring structure-property relationships using domain modeling. (a) To exploring relationships between structure and property of polymer materials, ${ }^{1} \mathrm{H}-$ static solid-state NMR (sSNMR) spectrum, primary structure information and physical property data obtained from TG, DTA and DTG were used. (b) Domain modeling was applied to the input ${ }^{1} \mathrm{H}$-static ssNMR spectrum of polymer materials to obtain domain structural information. (b) The domain proportion, primary structure data and thermophysical property data were integrated for data analysis such as statistical analysis, SOM and MBA. ${ }^{1} \mathrm{H}$-static sSNMR: proton static solid-state Nuclear Magnetic Resonance, TG: thermogravimetry, DTA: differential thermal analysis, DTG: derivative thermogravimetry, Tm: melting temperature, Td: thermal decomposition temperature, Tg: glass transition temperature, STFT: short-time Fourier transform, MBA: market basket analysis, SOM: self-organization map. 


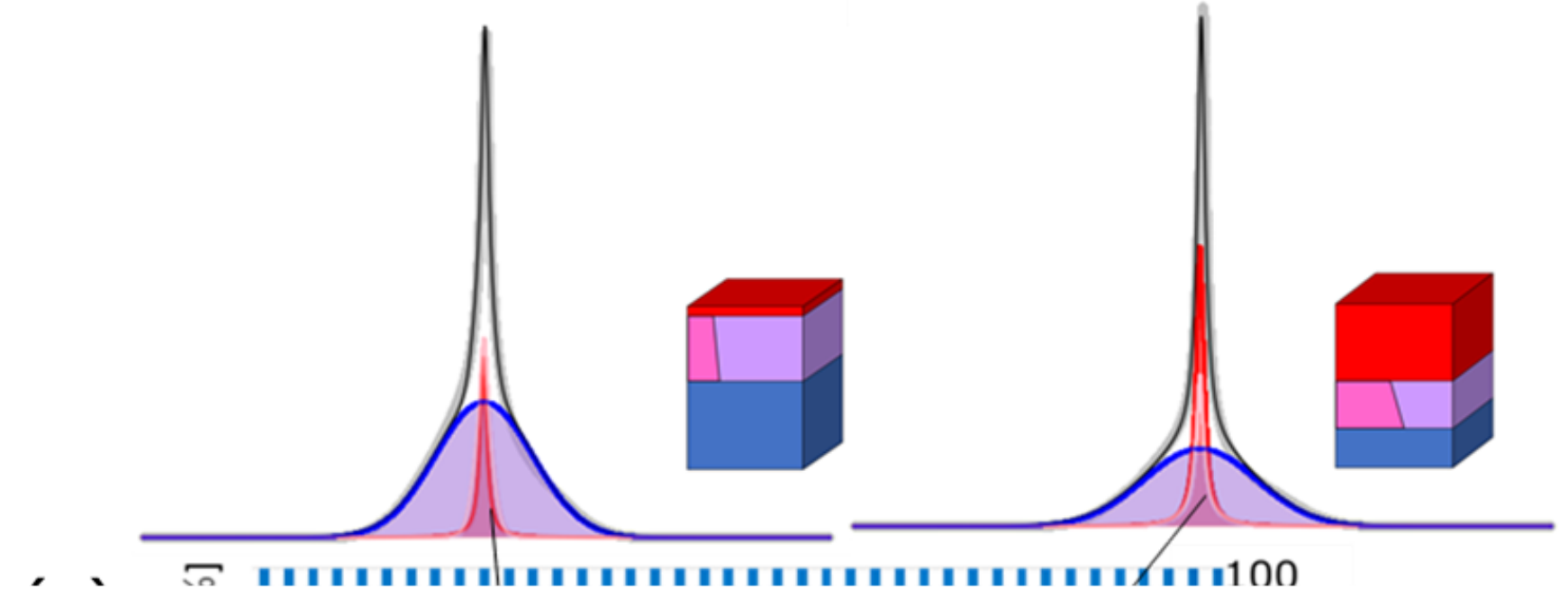

\section{Figure 2}

Results of domain proportion calculation of polymer materials. The ${ }^{1} \mathrm{H}$-static ssNMR spectra of 71 polymer materials were separated by domain modeling. Based on the results, the domain models are shown. (a) shows an example of $\mathrm{PHBH}$, (b) shows an example of PCL, and (c) shows the domain ratios of 71 polymer materials. Polymer sample ID numbers in the figure refer to Table S2. Red囚Mobile, Magenta囚Intermediate(Mobile), Violet囚Intermediate(Rigid), Blue囚Rigid. The calculated distributions of $T_{2}$ 
relaxation time and thermophysical properties of the domain components of each polymer are shown (Figure 3). The box-and-whisker plot shows that the average $T_{2}$ relaxation time information is $0.96 \mathrm{~ms}$ for Mobile, $0.55 \mathrm{~ms}$ for Intermediate (Mobile), $0.32 \mathrm{~ms}$ for Intermediate (Rigid), and $0.11 \mathrm{~ms}$ for Rigid. The weighted average (WA) of the polymer materials showed a distribution among the samples, among which PCL (Figure3, red squares) was high and PHBH (Figure 3, yellow circles) was low. The same was true for the results of thermal analysis spectral data (Table S3). Based on the domain component ratios, the estimated domain ratio diagrams were inserted for the highest PCL and lowest PHBH of WA.

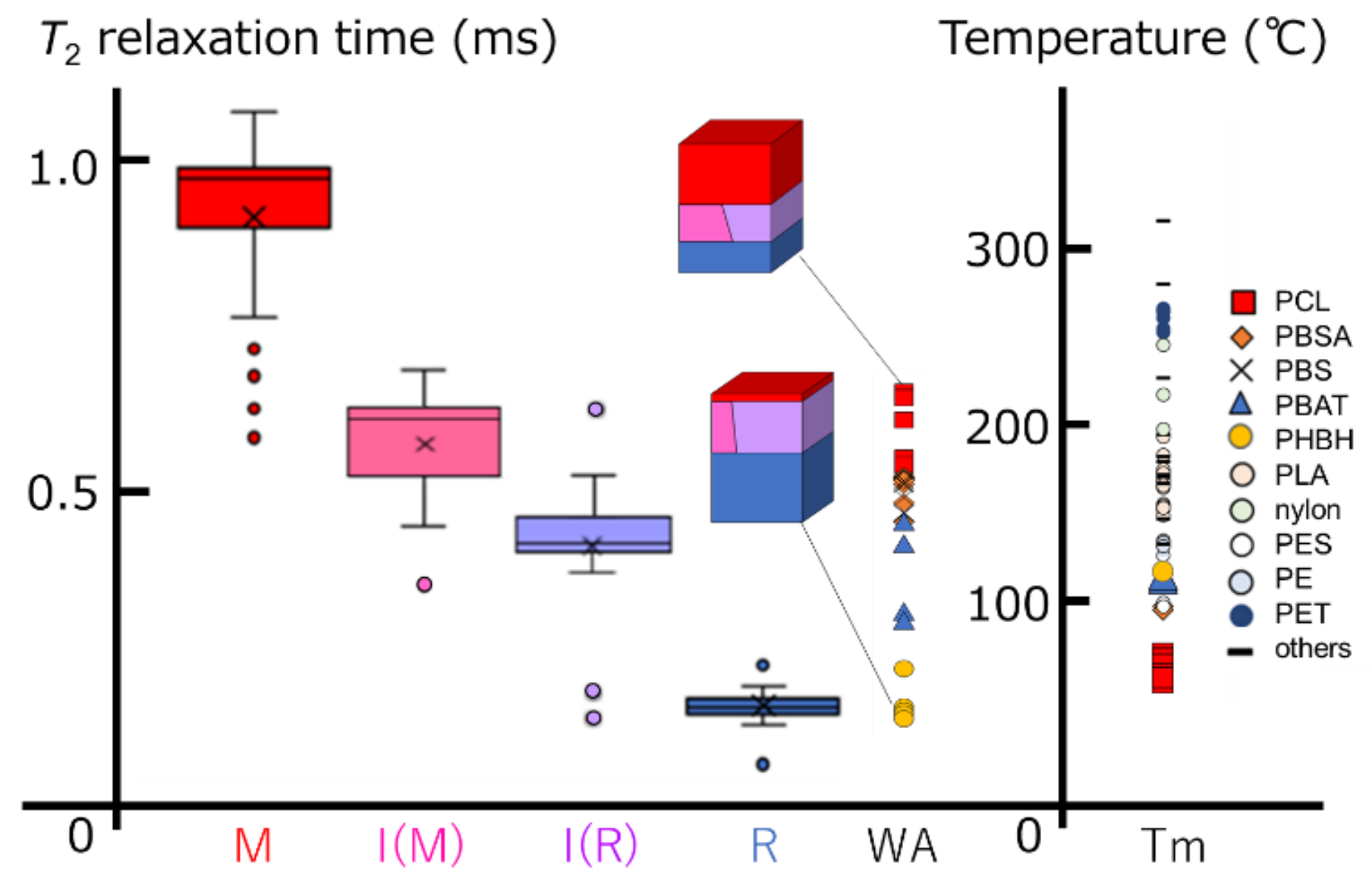

Figure 3

Diagram of polymer properties with $T_{2}$ relaxation time among four domain components and melting temperature. M: Red box plot; Mobile, I(M): Pink box plot; Intermediate (Mobile), I(R): Violet box plot; Intermediate (Rigid), R: Blue box plot; Rigid, WA: Weighted average, $T_{\mathrm{m}}$ : Melting temperature. $\mathrm{x}$ : Average $T_{2}$ relaxation time. The box and whisker plot displays the minimum, first quartile, median, third quartile, maximum, and outliers. The estimated domain proportion models were inserted for the highest PCL and lowest $\mathrm{PHBH}$ of WA.

Figure 4 
Result of SOM. Visualization of 3D SOM compressed into 2D SOM with $4 \times 6$ segments, red: positive, blue: negative. red square: PCL, orange: PBSA, black cross: PBS, blue triangle: PBAT, yellow circle: PHBH, light pink: PLA, light green: nylon, white: PES, light blue: $\mathrm{PE}$, navy blue: $\mathrm{PET}$, black bar: other polymers. Polymer numbers in the figure refer to Table $\mathrm{S} 1$.

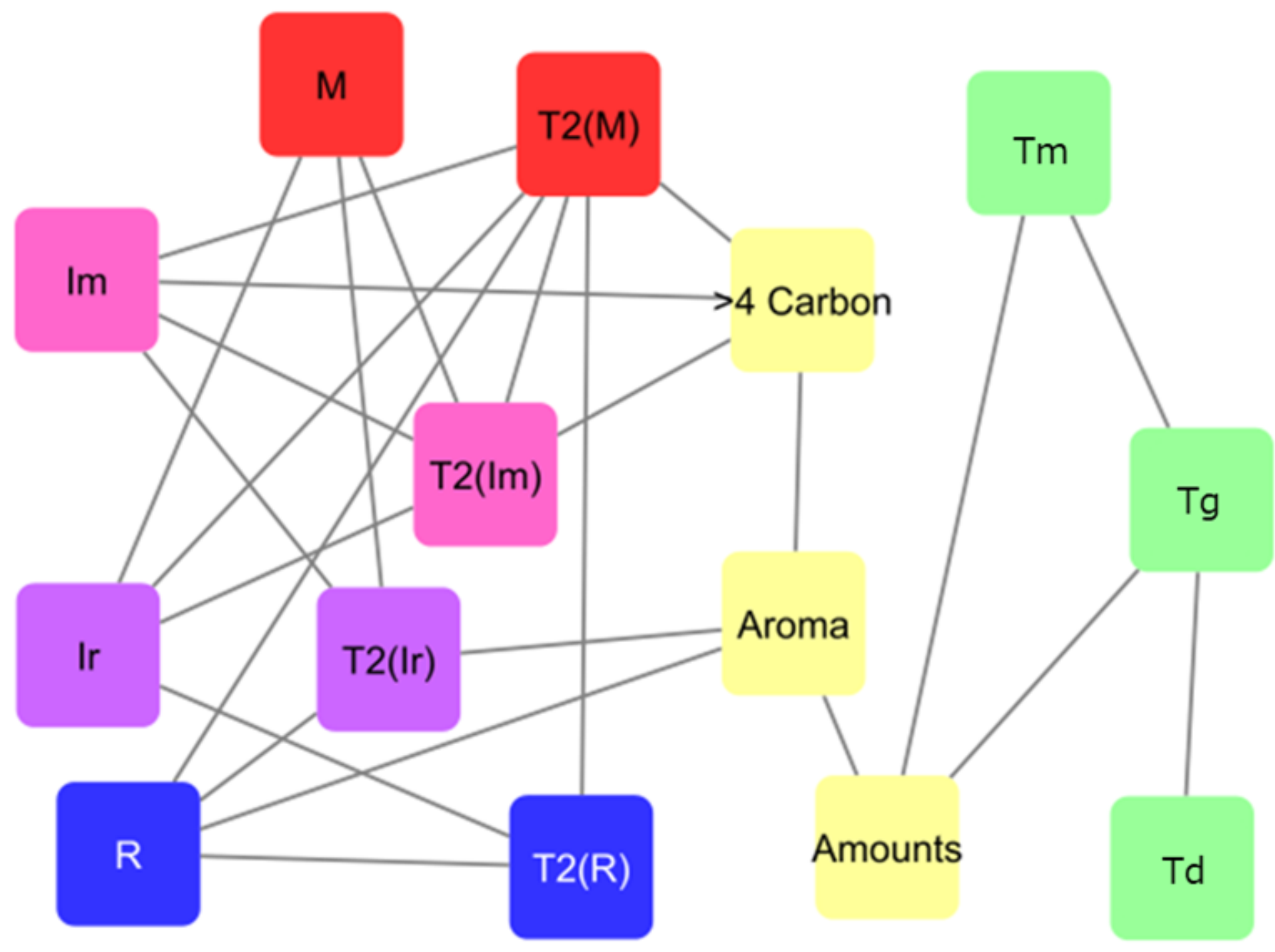

\section{Figure 5}

Selected MBA network for $T_{2}$ relaxation time data. From the results of the market basket analysis, only the relationships between the simulated domain structure information and $T_{2}$ relaxation time data, experimental melting temperature ( $\mathrm{Tm})$, thermal decomposition temperature ( $\mathrm{Td})$, glass transition temperature $(\mathrm{Tg})$, and data on primary structure were extracted. M: Mobile domain, Im: MAPE Filterderived Intermediate domain (Intermediate (Mobile)), Ir: DQ Filter-derived Intermediate domain (Intermediate (Rigid)), R: Rigid domain, T2(M): $T_{2}$ relaxation time information for mobile domain, T2(Im): $T_{2}$ relaxation time information for Intermediate (Mobile) domain, T2(R): $T_{2}$ relaxation time information for rigid domain, T2(Ir): $T_{2}$ relaxation time information for Intermediate (Rigid) domain, $>4$ carbon: linearly connected methylenes $>4$ carbons, Aroma: aromaticity, Amounts: sample amounts. 


\section{Supplementary Files}

This is a list of supplementary files associated with this preprint. Click to download.

- Supportinginformation.docx 